\title{
Relativistic collective coordinate quantization of solitons: Spinning Skyrmion
}

\section{$\operatorname{AUTHOR}(\mathrm{S})$ :}

Hata, Hiroyuki; Kikuchi, Toru

\section{CITATION:}

Hata, Hiroyuki ... [et al]. Relativistic collective coordinate quantization of solitons: Spinning Skyrmion. Physical Review D 2010, 82(2): 025017.

\section{ISSUE DATE:}

2010-07

URL:

http://hdl.handle.net/2433/128848

RIGHT:

(C) 2010 The American Physical Society 
PHYSICAL REVIEW D 82, 025017 (2010)

\title{
Relativistic collective coordinate quantization of solitons: Spinning Skyrmion
}

\author{
Hiroyuki Hata* and Toru Kikuchi ${ }^{\dagger}$ \\ Department of Physics, Kyoto University, Kyoto 606-8502, Japan
}

(Received 5 March 2010; published 27 July 2010)

\begin{abstract}
We develop a consistent relativistic generalization of collective coordinate quantization of field theory solitons. Our principle of introducing collective coordinates is that the equations of motion of the collective coordinates ensure those of the original field theory. We illustrate this principle with the quantization of spinning degrees of freedom of Skyrmion representing baryons. We calculate the leading relativistic corrections to the static properties of nucleons, and find that the corrections are non-negligible ones of $10 \%$ to $20 \%$.
\end{abstract}

DOI: 10.1103/PhysRevD.82.025017

PACS numbers: 12.39.Dc, 11.10.-z, 14.20.Dh

\section{INTRODUCTION}

Collective coordinates of a field theory soliton describe the motion of the soliton in the symmetry directions of the field theory action. They correspond to the zero modes around the soliton and are important in understanding its low energy dynamics. The simplest way of introducing the collective coordinates is to promote the (originally constant) parameters of a static soliton to time-dependent dynamical variables by discarding the fluctuation of nonzero modes. This is valid in the nonrelativistic cases where time derivatives of the collective coordinates are small enough.

However, in certain circumstances, it is inappropriate to assume that solitons move slowly; relativistic corrections come to be important. A typical example is the spinning Skyrmion representing baryons [1]. In the standard quantization of the rotational collective coordinate of the Skyrmion $[2,3]$, the mass of the baryons is given as the sum of the energy of the static classical solution and the rotational kinetic energy of a spherical rigid body. For the nucleon (delta), about $8 \%(30 \%)$ of the total mass comes from the rotational energy. This leads to the picture of a baryon rotating with a velocity comparable to the light velocity at the radius of order $1 \mathrm{fm}$. Since the baryon rotates so fast, it is natural to expect that the baryon cannot maintain its original spherical shape. The collective coordinate of rotation should be introduced in a consistent way that can express the deformation.

The purpose of this paper is, on the basis of a general and simple principle of introducing the collective coordinates, to carry out the quantization of rotational degrees of freedom of the $S U(2)$ Skyrmion to evaluate the relativistic corrections to the static properties of nucleons. While several authors have discussed the deformation of spinning Skyrmions with various physical pictures (see Refs. [4-8] for earlier works), we emphasize that the deformation is naturally induced in our treatment of collective coordi-

\footnotetext{
*hata@gauge.scphys.kyoto-u.ac.jp

${ }^{\dagger}$ kikuchi@gauge.scphys.kyoto-u.ac.jp
}

nates. Another feature of our treatment of a rotational collective coordinate is that we introduce it through coordinate-transformed static solitons with a new coordinate depending on the time derivative of the collective coordinate [see Eq. (5) together with (6)].

\section{GENERAL PRINCIPLE}

Our basic idea is to avoid mismatch between the field theory dynamics and the collective coordinate dynamics. While the system of collective coordinates has a finite number of degrees of freedom, the original field theory has an infinite one. Therefore, even when the equation of motion (EOM) of collective coordinates holds, it does not necessarily mean that the soliton configuration satisfies its field theory EOM. Then our general principle of introduction of collective coordinates is simply stated as follows: Collective coordinates must be introduced in such a way that the EOM of the collective coordinates ensures that of the original field theory. If the original field theory is a relativistic one, this principle should automatically lead to a relativistic dynamics of collective coordinates.

One way to realize this principle is, starting with suitably introduced collective coordinates, to integrate over the nonzero modes around the soliton. Namely, we solve the EOM of the nonzero modes to express them in terms of the collective coordinates. Since the field theory EOM is equivalent to the set of EOMs of both the zero and the nonzero modes, solving the EOM for the latter should lead to a system of collective coordinates satisfying our principle. In fact, the relativistic energy of the center-of-mass motion, $E=\sqrt{P^{2}+M^{2}}$, is obtained by this method in scalar field theories in $1+1$ dimensions $[9,10]$. However, no explicit calculation by this method has been carried out for more complicated cases other than the center of mass, in particular, for the collective coordinate of rotational motion.

In this paper, we propose another way: First, the collective coordinates are put into the static classical solution in such a way as to fulfill our principle of EOMs. Then, the (relativistic) Lagrangian of the collective coordinates is 
obtained simply by inserting this soliton field into the field theory Lagrangian density and carrying out the space integration. In this process, nonzero modes do not appear; in other words, the EOM of nonzero modes implies that they are equal to zero in this framework.

In the case of the collective coordinate $\boldsymbol{X}(t)$ of the center of mass of a soliton in a scalar field theory, we can show that our principle is satisfied, up to $O\left(\partial_{0}^{6}\right)$ terms, by the relativistic replacement of the space coordinate $x$ of the static soliton $\varphi^{\mathrm{cl}}(\boldsymbol{x})$ by $(\boldsymbol{x}-\boldsymbol{X}(t)) / \sqrt{1-\boldsymbol{V}^{2}}$ with $\boldsymbol{V}=\dot{\boldsymbol{X}}$, leading to the relativistic Lagrangian $L=-M \sqrt{1-V^{2}}$. On the other hand, it is a nontrivial task to realize our principle for the collective coordinate of space rotation. In the rest of this paper, we illustrate our general method with two-flavor spinning Skyrmion to obtain the relativistic corrections to the earlier results $[2,3]$. Our finding here is that our principle of EOMs can be satisfied, up to $O\left(\partial_{0}^{4}\right)$ terms, by taking the coordinate-transformed static soliton given by (5) and (6) with suitably chosen functions $A(r)$, $B(r)$, and $C(r)$.

Although we focus on the spinning collective coordinate of the Skyrmion in the rest of this paper, we wish to emphasize that our method of extracting the system of collective coordinates on the basis of the principle of EOMs is simpler and has wider applicability since we are not bothered by the nonzero modes. In addition, our method is interesting also in that we can directly know how the soliton deforms due to fast collective motion, since the collective coordinates are introduced by deforming the coordinate of the static solution.

\section{THE SKYRMION}

The $S U(2)$ Skyrme model [1] is described by the chiral Lagrangian with the Skyrme term:

$$
\mathcal{L}=\operatorname{tr}\left\{-\frac{f_{\pi}^{2}}{16} L_{\mu}^{2}+\frac{1}{32 e^{2}}\left[L_{\mu}, L_{\nu}\right]^{2}+\frac{f_{\pi}^{2}}{8} m_{\pi}^{2}\left(U-1_{2}\right)\right\}
$$

where $U(x)$ is an $S U(2)$ matrix and $L_{\mu}=-i U \partial_{\mu} U^{\dagger}$. The EOM reads

$$
\partial_{\mu}\left(L^{\mu}-\frac{1}{e^{2} f_{\pi}^{2}}\left[L_{\nu},\left[L^{\mu}, L^{\nu}\right]\right]\right)-i m_{\pi}^{2}\left(U-\frac{1}{2} \operatorname{tr} U\right)=0 .
$$

This theory has a static soliton solution $U^{\mathrm{cl}}(\boldsymbol{x})=\exp (i \boldsymbol{x}$. $\tau F(r) / r$ ) called Skyrmion, where $\tau_{i}$ are sigma matrices and $F$ is a function of $r=|x|$. This static solution has a rotational collective coordinate, that is, $U^{\mathrm{cl}}\left(R^{-1} \boldsymbol{x}\right)$ is also an solution for any time-independent $S O(3)$ matrix $R$. For the Skyrmion, the space rotation is equivalent to the isospin rotation; $U^{\mathrm{cl}}\left(R^{-1} \boldsymbol{x}\right)=W U^{\mathrm{cl}}(\boldsymbol{x}) W^{-1}$ with $W$ being the $S U(2)$ matrix corresponding to $R$.

The standard way $[2,3]$ to quantize these spinning degrees of freedom is as follows: Promoting the constant
$S O(3)$ matrix $R$ [or equivalently, the $S U(2)$ matrix $W$ ] to a time-dependent one, we take

$$
U(\boldsymbol{x}, t)=U^{\mathrm{cl}}\left(R^{-1}(t) \boldsymbol{x}\right),
$$

as the spinning Skyrmion field and insert it into the Lagrangian density (1). Carrying out the spatial integration, we get the Lagrangian of $R(t), L(R, \dot{R})=$ $\int d^{3} x \mathcal{L}\left(U(\boldsymbol{x}, t)=U^{\mathrm{cl}}\left(R^{-1}(t) \boldsymbol{x}\right)\right)=-M^{\mathrm{cl}}+\frac{1}{2} I \boldsymbol{\Omega}^{2}$, where the rest mass $M^{\mathrm{cl}}$ and the moment of inertia $I$ are functionals of $F(r)$, and $\boldsymbol{\Omega}$ is the angular velocity $\Omega_{i}=$ $\frac{1}{2} \varepsilon_{i j k}\left(R^{-1} \dot{R}\right)_{j k}=\operatorname{tr}\left(i \dot{W} W^{-1} \tau_{i}\right)$ (precisely speaking, $\boldsymbol{\Omega}$ is the angular velocity in isospace). Note that $\boldsymbol{\Omega}^{2}=$ $-\frac{1}{2} \operatorname{Tr}\left(R^{-1} \dot{R}\right)^{2}$. We carry out the quantization of the dynamical variable $R$ using this Lagrangian.

This quantization procedure is evidently a nonrelativistic one since the Lagrangian of $R$ is simply that of a rigid body. In addition, the field theory EOM (2) with $U$ given by (3) is violated by the $O\left(\mathbf{\Omega}^{2}\right)$ term even if we use the EOM of $R$,

$$
(d / d t) \boldsymbol{\Omega}=0 .
$$

Moreover, the relativistic corrections to the various properties of baryons seem to be important as we explained in the Introduction.

\section{DEFORMATION OF SPINNING SKYRMION}

We wish to give a relativistic version of the spinning Skyrmion field (3) on the basis of our general principle of introducing the collective coordinates. However, it is difficult to find the complete one in a closed form. Instead, in this paper we present the leading correction to the rigid body approximation (3) with respect to the power of the angular velocity $\boldsymbol{\Omega}$, or equivalently, the number of time derivatives. Our spinning Skyrmion takes the form

$$
U(\boldsymbol{x}, t)=U^{\mathrm{cl}}(\boldsymbol{y}),
$$

with $\boldsymbol{y}$ given by

$$
\begin{aligned}
\boldsymbol{y}= & \left(1+A(r)\left(\dot{R} R^{-1} \boldsymbol{x}\right)^{2}+r^{2} B(r) \operatorname{Tr}\left(R^{-1} \dot{R}\right)^{2}\right) R^{-1} \boldsymbol{x} \\
& +r^{2} C(r)\left[\left(R^{-1} \dot{R}\right)^{2} R^{-1} \boldsymbol{x}\right],
\end{aligned}
$$

where the functions $A, B$, and $C$ are to be determined to fulfill our principle concerning the EOMs. The form of $\boldsymbol{y}$ is the most general one which is at most quadratic in $\boldsymbol{\Omega}$, odd under $\boldsymbol{x} \rightarrow-\boldsymbol{x}$, and has the property that the left (right) constant $S O(3)$ transformation on $R$ induces the space (isospin) rotation. The last property is the basic one for the quantization of Skyrmion. It can easily be seen that the field configuration (5) with $\boldsymbol{y}$ of (6) represents a spheroidal one.

\section{DETERMINATION OF $(A, B, C)$}

For determining $A, B$, and $C$ from our principle, we substitute $U(\boldsymbol{x}, t)$ of (5) into the field theory EOM (2) to 
find that its left-hand side is, upon using the EOM of $U^{\mathrm{cl}}$, given by

$$
\begin{aligned}
& {\left[\left(\frac{d}{d t} R^{-1} \dot{R}\right) \boldsymbol{y}\right]_{i}\left(L_{i}^{\mathrm{cl}}-\frac{1}{e^{2} f_{\pi}^{2}}\left[L_{j}^{\mathrm{cl}},\left[L_{i}^{\mathrm{cl}}, L_{j}^{\mathrm{cl}}\right]\right]\right)} \\
& \quad+r^{2} \operatorname{Tr}\left(R^{-1} \dot{R}\right)^{2}(\boldsymbol{y} \cdot \boldsymbol{\tau}) \times \mathrm{EQ}_{1}+\left(R^{-1} \dot{R} \boldsymbol{y}\right)^{2}(\boldsymbol{y} \cdot \boldsymbol{\tau}) \\
& \quad \times \mathrm{EQ}_{2}+r^{2}\left[\left(R^{-1} \dot{R}\right)^{2} \boldsymbol{y}\right] \cdot \boldsymbol{\tau} \times \mathrm{EQ}_{3} \\
& \quad+r\left[\boldsymbol{y} \times\left(R^{-1} \dot{R}\right)^{2} \boldsymbol{y}\right] \cdot \boldsymbol{\tau} \times \mathrm{EQ}_{4}+O\left(\partial_{0}^{4}\right),
\end{aligned}
$$

with $L_{i}^{\mathrm{cl}}=-i U^{\mathrm{cl}}(\boldsymbol{y})\left(\partial / \partial y_{i}\right) U^{\mathrm{cl}}(\boldsymbol{y})^{\dagger} . \mathrm{EQ}_{i}(i=1, \ldots, 4)$ are linear in $(A, B, C)$ and their first and second derivatives with respect to $r$ with coefficients given in terms of $F$ and its derivatives. Concrete expressions of $\mathrm{EQ}_{i}$ are very lengthy, and they are given in the Appendix. Here, we present a special linear combination of $\mathrm{EQ}_{i}$ which consists of only a special combination $Y=-A+3 B+C$ and its derivatives:

$$
\begin{aligned}
\mathrm{EQ}_{Y}= & -3 \mathrm{EQ}_{1}+\mathrm{EQ}_{2}-\mathrm{EQ}_{3} \\
= & \left(1+\frac{8}{e^{2} f_{\pi}^{2}} \frac{\sin ^{2} F}{r^{2}}\right) F^{\prime} \frac{d^{2} Y}{d r^{2}}+\left\{2 F^{\prime \prime}+\frac{8}{r} F^{\prime}+\frac{8}{e^{2} f_{\pi}^{2}}\left(2 \frac{\sin ^{2} F}{r^{2}} F^{\prime \prime}+\frac{\sin 2 F}{r^{2}}\left(F^{\prime}\right)^{2}+\frac{6}{r} \frac{\sin ^{2} F}{r^{2}} F^{\prime}\right)\right\} \frac{d Y}{d r} \\
& +\left\{\frac{6}{r} F^{\prime \prime}+\frac{14}{r^{2}} F^{\prime}-\frac{2}{r^{3}} \sin 2 F+\frac{8}{e^{2} f_{\pi}^{2}}\left(\frac{8}{r} \frac{\sin ^{2} F}{r^{2}} F^{\prime \prime}+\frac{4}{r^{3}} \sin 2 F\left(F^{\prime}\right)^{2}+\frac{6}{r^{2}} \frac{\sin ^{2} F}{r^{2}} F^{\prime}-\frac{2}{r^{3}} \frac{\sin ^{2} F}{r^{2}} \sin 2 F\right)\right\} Y \\
& -\frac{1}{2 r^{3}} \sin 2 F+\frac{2}{e^{2} f_{\pi}^{2}}\left(\frac{2}{r} \frac{\sin ^{2} F}{r^{2}} F^{\prime \prime}+\frac{1}{r^{3}} \sin 2 F\left(F^{\prime}\right)^{2}+\frac{4}{r^{2}} \frac{\sin ^{2} F}{r^{2}} F^{\prime}-\frac{2}{r^{3}} \sin 2 F \frac{\sin ^{2} F}{r^{2}}\right),
\end{aligned}
$$

where the prime on $F$ denotes an $r$ derivative. The function $Y(r)$ is related to the angle average of $\boldsymbol{y}^{2}$ with respect to $\boldsymbol{x}$, $1 /(4 \pi) \int d \Omega_{x} y^{2}=r^{2}\left(1-\frac{4}{3} r^{2} \boldsymbol{\Omega}^{2} Y(r)\right)$, and it seems to represent an independent degree of freedom of the deformation due to spinning.

Returning to (7), our principle of introducing the collective coordinates demands that (7) vanish identically upon using the EOM of $R$. As we will see later, the EOM of $R$ remains unchanged from (4) even if the relativistic corrections are introduced. This implies that the three functions $(A, B, C)$ must satisfy four differential equations $\mathrm{EQ}_{i}=0$ $(i=1, \ldots, 4)$, which is apparently overdetermined. Fortunately, $\mathrm{EQ}_{3}$ and $\mathrm{EQ}_{4}$ are not independent; we have $\mathrm{EQ}_{4}=-\tan F \mathrm{EQ}_{3}$ [see Eqs. (A3) and (A4)]. Therefore, $(A, B, C)$ are determined by three inhomogeneous linear differential equations of second order, $\mathrm{EQ}_{i}=0(i=1,2$, 3 ), or another independent set $\mathrm{EQ}_{2}=\mathrm{EQ}_{3}=\mathrm{EQ}_{Y}=0$ with $\mathrm{EQ}_{Y}$ given by (8). The boundary conditions for $(A, B, C)$ at $r=0$ and $r=\infty$ are chosen to be the least singular ones among those allowed by the differential equations; $\quad(A, B, C) \sim\left(1,1 / r^{2}, 1 / r^{2}\right) \quad$ as $\quad r \rightarrow 0 \quad$ and $(A, B, C-A) \sim\left(1 / r, 1 / r^{3}, 1 / r^{2}\right)$ as $r \rightarrow \infty$, both up to numerical coefficients. By this choice, relativistic corrections to various physical quantities of baryons, which are given as space integrations with integrands consisting of $(A, B, C)$ and $F$ and their derivatives, are unambiguously determined.

\section{LAGRANGIAN OF $R$}

Inserting the spinning field $U(\boldsymbol{x}, t)$ of (5) into the Lagrangian density (1) and spatially integrating it, we obtain the Lagrangian of $R$ of the following form:

$$
L=-M^{\mathrm{cl}}+\frac{1}{2} \mathcal{I} \boldsymbol{\Omega}^{2}+\frac{1}{4} \mathcal{J} \boldsymbol{\Omega}^{4},
$$

where $I$ and $M^{\mathrm{cl}}$ are the same as in the rigid body approximation [3], and the last $\boldsymbol{\Omega}^{4}$ term represents our relativistic correction. This Lagrangian should be regarded as the rotational motion counterpart of the relativistic Lagrangian of center of mass; $-M \sqrt{1-V^{2}}=-M+$ $\frac{1}{2} M V^{2}+\frac{1}{8} M V^{4}+\cdots$. The EOM of $R$ derived from (9) is

$$
\frac{d}{d t}\left[\left(I+\mathcal{J} \boldsymbol{\Omega}^{2}\right) \boldsymbol{\Omega}\right]=0 .
$$

This implies (4), which we already used in deriving the differential equations for $(A, B, C)$.

The coefficient $\mathcal{J}$ of the relativistic correction term has two origins, $\mathcal{J}=\mathcal{J}_{1}+\mathcal{J}_{2} ; \mathcal{J}_{1}$ is from the part of the Skyrme model Lagrangian (1) quadratic in $L_{0}$ and hence is linear in $(A, B, C)$, while $\mathcal{J}_{2}$ is from the part of (1) without $L_{0}$ and is quadratic in $(A, B, C)$. The calculation of $\mathcal{J}_{2}$ is complicated, but fortunately we have the relation $\mathcal{J}_{1}+$ $2 \mathcal{J}_{2}=0$. This is understood by making the replacement $(A, B, C) \rightarrow \lambda(A, B, C)$ in (6) and using the fact that $\lambda=1$ must give an extremal of $L$ for any constant $\boldsymbol{\Omega}$ satisfying the EOM (4) due to our principle of introducing the collective coordinates and hence of determining $(A, B, C)$. Therefore, we have $\mathcal{J}=\mathcal{J}_{1} / 2$, which is explicitly given by

$$
\begin{aligned}
\mathcal{J}= & \frac{4 \pi f_{\pi}^{2}}{15} \int_{0}^{\infty} d r r^{4} \sin ^{2} F\left\{r Z^{\prime}+5 Z-C\right. \\
& +\frac{4}{e^{2} f_{\pi}^{2}}\left[\frac{\sin ^{2} F}{r^{2}}\left(r Z^{\prime}+3 Z+2 C\right)\right. \\
& \left.\left.-\left(F^{\prime}\right)^{2}\left(r Z^{\prime}+Z+C\right)\right]\right\},
\end{aligned}
$$

with $Z=-2 A+5 B+2 C$. 
Several comments are in order: First, the moment of inertia $I$ in the Lagrangian (9) receives no correction from the deformation of (6) as we mentioned before. A possible correction to $I$ is from the part of (1) without $L_{0}$ and is linear in $(A, B, C)$. The vanishing of this correction is shown by the same $\lambda$-rescaling argument as we used in deriving $\mathcal{J}_{1}+2 \mathcal{J}_{2}=0$. By the same reason, further correction to $\boldsymbol{y}(6)$ of $O\left(\partial_{0}^{4}\right)$ does not affect $\mathcal{J}$.

Our second comment is on another way of obtaining the expression (11) for $\mathcal{J}$. The isospin charge derived from (9) is

$$
I_{a}=\left(I+\mathcal{J} \mathbf{\Omega}^{2}\right) \Omega_{a} .
$$

On the other hand, $I_{a}$ is also given by $I_{a}=\int d^{3} x J_{V, a}^{\mu=0}$, where $J_{V, a}^{\mu}$ is the Noether current of $S U(2)_{V}$ symmetry derived from the Lagrangian density (1). By comparing the two expressions of $I_{a}$, we can directly obtain (11) from the Noether current. We can show in general that conserved charges derived from the Lagrangian (9) of the $R$ system and the corresponding ones derived as the space integration of the time component of the Noether currents in the Skyrme model agree with each other up to the EOM of $R$ and that in the Skyrme model.

Finally, if the pion mass $m_{\pi}$ in (1) is zero, the integration (11) for $\mathcal{J}$ diverges at $r=\infty$. This is the case also for relativistic corrections to other physical quantities. It is crucial for our analysis to introduce nonzero pion mass.

\section{STATIC PROPERTIES OF NUCLEONS}

The Hamiltonian of $R$ obtained from the Lagrangian (9) is given by

$$
H=M^{\mathrm{cl}}+\frac{1}{2} \mathcal{I} \boldsymbol{\Omega}^{2}+\frac{3}{4} \mathcal{J} \mathbf{\Omega}^{4} .
$$

For obtaining the value of $H$ for an eigenstate of the isospin, we have to solve

$$
\boldsymbol{I}^{2}=\left(I+\mathcal{J} \boldsymbol{\Omega}^{2}\right)^{2} \boldsymbol{\Omega}^{2},
$$

to express $\boldsymbol{\Omega}^{2}$ in terms of a given $\boldsymbol{I}^{2}$.

Following [3], we evaluated $f_{\pi}$ and $e$ in the Skyrme Lagrangian (1) by taking the masses of nucleon, $\Delta$ and pion $\quad\left(m_{N}=939 \mathrm{MeV}, \quad m_{\Delta}=1232 \mathrm{MeV}, \quad m_{\pi}=\right.$ $138 \mathrm{MeV}$ ) as inputs. Our result is $f_{\pi}=125 \mathrm{MeV}$ and $e=$ 5.64. Compared with the experimental value $f_{\pi}=$ $186 \mathrm{MeV}$, our $f_{\pi}$ is fairly improved from that of [3], $f_{\pi}=$ $108 \mathrm{MeV}$, without relativistic correction. We have also obtained the expressions of various static properties of nucleons with relativistic correction and computed their numerical values. For example, the isoscalar mean square charge radius is given by $\left\langle r^{2}\right\rangle_{I=0}=4 \pi \int_{0}^{\infty} d r r^{4} J_{B}^{\mathrm{cl} 0}(r) \times$ $\left(1+\frac{4}{3} \boldsymbol{\Omega}^{2} r^{2} Y(r)\right)$, with $J_{B}^{\mathrm{cl} 0}(r)=-1 /\left(2 \pi^{2}\right)(\sin F / r)^{2} \times$ $(d F / d r)$ being the baryon number density of the static Skyrmion $U^{\mathrm{cl}}(\boldsymbol{x})$. Our numerical results are summarized in Table I.
TABLE I. The static properties of nucleons. Prediction of this paper and that of Ref. [3] both use the experimental values of $\left(m_{N}, m_{\Delta}, m_{\pi}\right)$ as inputs. We follow the notations of Ref. [2].

\begin{tabular}{lccc}
\hline \hline & $\begin{array}{c}\text { Prediction } \\
\text { (this paper) }\end{array}$ & $\begin{array}{c}\text { Prediction } \\
\text { (Ref. [3]) }\end{array}$ & Experiment \\
\hline$f_{\pi}$ & $125 \mathrm{MeV}$ & $108 \mathrm{MeV}$ & $186 \mathrm{MeV}$ \\
\hline$\left\langle r^{2}\right\rangle_{I=0}^{1 / 2}$ & $0.59 \mathrm{fm}$ & $0.68 \mathrm{fm}$ & $0.81 \mathrm{fm}$ \\
$\left\langle r^{2}\right\rangle_{I=1}^{1 / 2}$ & $1.17 \mathrm{fm}$ & $1.04 \mathrm{fm}$ & $0.94 \mathrm{fm}$ \\
$\left\langle r^{2}\right\rangle_{M, I=0}^{1 / 2}$ & $0.85 \mathrm{fm}$ & $0.95 \mathrm{fm}$ & $0.82 \mathrm{fm}$ \\
$\left\langle r^{2}\right\rangle_{M, I=1}^{1 / 2}$ & $1.17 \mathrm{fm}$ & $1.04 \mathrm{fm}$ & $0.86 \mathrm{fm}$ \\
$\mu_{p}$ & 1.65 & 1.97 & 2.79 \\
$\mu_{n}$ & -0.99 & -1.24 & -1.91 \\
$\left|\mu_{p} / \mu_{n}\right|$ & 1.67 & 1.59 & 1.46 \\
$g_{A}$ & 0.58 & 0.65 & 1.24 \\
\hline \hline
\end{tabular}

As seen from the table, our relativistic correction is a non-negligible one of roughly $10 \%$ to $20 \%$ for every static property. (Note that each of our numerical values is not given simply by adding the $\boldsymbol{\Omega}^{2}$ correction to the value of Ref. [3], since the parameters $f_{\pi}$ and $e$ themselves are also changed.) Unfortunately, the correction is not in the direction of making the theoretical prediction closer to the experimental value for most of the quantities. However, we emphasize that this is not a problem of our basic principle of collective coordinate quantization; it might be due to taking only the first term of the expansion in powers of $\boldsymbol{\Omega}^{2}$, or to the fact that the Skyrme model is merely an approximation to QCD. In relation to the first possibility, the ratio of the contributions of the three terms of the Hamiltonian (13) to the baryon mass is approximately $89: 7: 4$ for the nucleon, while it is $68: 14: 18$ for $\Delta$. This shows that our analysis using $\Delta$ needs better treatment of the relativistic correction beyond a simple expansion in powers of angular velocity.

\section{SUMMARY}

In this paper, we proposed a general principle of introducing collective coordinates of solitons and applied it to the quantization of spinning motion of Skyrmion. We computed the leading relativistic corrections to the Lagrangian of the rotational motion and various physical quantities of baryons. Compared with the rigid body approximation, the value of the decay constant $f_{\pi}$ has become fairly close to the experimental one due to the correction, but the numerical results are not good for other static properties of nucleons. Putting aside the problem of comparison with the experimental values, our result shows the importance of relativistic treatment of the spinning collective coordinate beyond the rigid body approximation.

Finally, application of our principle of collective coordinate quantization to other interesting physical systems is of course an important future subject. 


\section{ACKNOWLEDGMENTS}

We would like to thank Kenji Fukushima, Koji Hashimoto, Antal Jevicki, and Keisuke Ohashi for valuable discussions. The work of H. H. was supported in part by a Grant-in-Aid for Scientific Research (C) No. 21540264 from the Japan Society for the Promotion of Science (JSPS). The work of T. K. was supported by a Grant-inAid for JSPS Fellows No. 21-951. The numerical calculations were carried out on Altix3700 BX2 at YITP in
Kyoto University. This work was also supported by the Grant-in-Aid for the Global COE Program "The Next Generation of Physics, Spun from Universality and Emergence" from the Ministry of Education, Culture, Sports, Science and Technology (MEXT) of Japan.

\section{APPENDIX: EXPLICIT EXPRESSIONS OF EQ $\mathbf{Q}_{1,2,3,4}$}

In this Appendix, we present concrete expressions of the four quantities $\mathrm{EQ}_{i}(i=1,2,3,4)$ appearing in (7):

$$
\begin{aligned}
\mathrm{EQ}_{1}= & \frac{2}{r^{2}} F^{\prime} A-F^{\prime} \frac{d^{2} B}{d r^{2}}-2\left(F^{\prime \prime}+\frac{4}{r} F^{\prime}\right) \frac{d B}{d r}-\frac{2}{r}\left(3 F^{\prime \prime}+\frac{7}{r} F^{\prime}-\frac{1}{r^{2}} \sin 2 F\right) B-\frac{1}{r^{2}}\left(2 F^{\prime}-\frac{1}{r} \sin 2 F\right) C \\
& +\frac{1}{f_{\pi}^{2} e^{2}}\left\{\frac{4}{r^{4}}(1-\cos 2 F) F^{\prime} A-\frac{4}{r^{2}}(1-\cos 2 F) F^{\prime} \frac{d^{2} B}{d r^{2}}-\frac{8}{r^{2}}\left[(1-\cos 2 F)\left(F^{\prime \prime}+\frac{3}{r} F^{\prime}\right)+\sin 2 F\left(F^{\prime}\right)^{2}\right] \frac{d B}{d r}\right. \\
& -\frac{8}{r^{3}}\left[(1-\cos 2 F)\left(4 F^{\prime \prime}+\frac{3}{r} F^{\prime}-\frac{1}{r^{2}} \sin 2 F\right)+4 \sin 2 F\left(F^{\prime}\right)^{2}\right] B-\frac{2}{r^{3}}(1-\cos 2 F) F^{\prime} \frac{d C}{d r} \\
& \left.-\frac{4}{r^{3}}\left[(1-\cos 2 F)\left(F^{\prime \prime}+\frac{2}{r} F^{\prime}-\frac{1}{r^{2}} \sin 2 F\right)+\sin 2 F\left(F^{\prime}\right)^{2}\right] C\right\}, \\
\mathrm{EQ}_{2}= & -F^{\prime} \frac{d^{2} A}{d r^{2}}-2\left(F^{\prime \prime}+\frac{4}{r} F^{\prime}\right) \frac{d A}{d r}+\frac{2}{r}\left[-3 F^{\prime \prime}+\frac{1}{r}(\cos 2 F-3) F^{\prime}+\frac{1}{r^{2}} \sin 2 F\right] A+\left(F^{\prime}-\frac{1}{2 r} \sin 2 F\right) \frac{d^{2} C}{d r^{2}} \\
& +\left[2 F^{\prime \prime}+\frac{1}{r}(7-\cos 2 F) F^{\prime}-\frac{3}{r^{2}} \sin 2 F\right] \frac{d C}{d r}+\frac{2}{r}\left[3 F^{\prime \prime}+\frac{2}{r}(1-\cos 2 F) F^{\prime}\right] C \\
& +\frac{1}{f_{\pi}^{2} e^{2}}\left\{\frac{2}{r^{3}}\left[(1-\cos 2 F)\left(F^{\prime \prime}+\frac{2}{r} F^{\prime}-\frac{2}{r^{2}} \sin 2 F\right)+2 \sin 2 F\left(F^{\prime}\right)^{2}\right]-\frac{4}{r^{2}}(1-\cos 2 F) F^{\prime} \frac{d^{2} A}{d r^{2}}\right. \\
& -\frac{4}{r^{2}}\left[2(1-\cos 2 F)\left(F^{\prime \prime}+\frac{3}{r} F^{\prime}\right)+\sin 2 F\left(F^{\prime}\right)^{2}\right] \frac{d A}{d r}-\frac{4}{r^{3}}\left[(1-\cos 2 F)\left(8 F^{\prime \prime}+\frac{1}{r}(1-2 \cos 2 F) F^{\prime}-\frac{2}{r^{2}} \sin 2 F\right)\right. \\
& \left.+5 \sin 2 F\left(F^{\prime}\right)^{2}\right] A+\frac{1}{r^{2}}(1-\cos 2 F)\left(4 F^{\prime}-\frac{1}{r} \sin 2 F\right) \frac{d^{2} C}{d r^{2}} \\
& +\frac{2}{r^{2}}\left[(1-\cos 2 F)\left(4 F^{\prime \prime}+\frac{1}{r}(7-2 \cos 2 F) F^{\prime}-\frac{2}{r^{2}} \sin 2 F\right)+2 \sin 2 F\left(F^{\prime}\right)^{2}\right] \frac{d C}{d r} \\
& \left.+\frac{4}{r^{3}}\left[(1-\cos 2 F)\left(5 F^{\prime \prime}-\frac{4}{r}(1+\cos 2 F) F^{\prime}+\frac{2}{r^{2}} \sin 2 F\right)+4 \sin 2 F\left(F^{\prime}\right)^{2}\right] C\right\},
\end{aligned}
$$

where $\mathrm{EQ}_{34}$ in (A3) and (A4) is given by

$$
\begin{aligned}
\mathrm{EQ}_{34}= & \frac{1}{2 r^{3}} \sin F+\frac{2}{r^{2}} \cos F F^{\prime} A-\frac{1}{2 r} \sin F \frac{d^{2} C}{d r^{2}}-\frac{1}{r}\left(\cos F F^{\prime}+\frac{3}{r} \sin F\right) \frac{d C}{d r}-\frac{1}{r^{2}}\left(4 \cos F F^{\prime}+\frac{1}{r} \sin F\right) C \\
& +\frac{1}{f_{\pi}^{2} e^{2}} \sin F\left\{\frac{2}{r^{3}}\left[\left(F^{\prime}\right)^{2}-\frac{1}{r^{2}}(1-\cos 2 F)\right]+\frac{4}{r^{2}}\left(F^{\prime}\right)^{2} \frac{d A}{d r}+\frac{4}{r^{3}} F^{\prime}\left(3 F^{\prime}+\frac{2}{r} \sin 2 F\right) A-\frac{1}{r^{3}}(1-\cos 2 F) \frac{d^{2} C}{d r^{2}}\right. \\
& \left.-\frac{4}{r^{2}}\left[\left(F^{\prime}\right)^{2}+\frac{1}{r} \sin 2 F F^{\prime}+\frac{1}{r^{2}}(1-\cos 2 F)\right] \frac{d C}{d r}-\frac{4}{r^{3}}\left[\left(F^{\prime}\right)^{2}+\frac{4}{r} \sin 2 F F^{\prime}-\frac{1}{r^{2}}(1-\cos 2 F)\right] C\right\} .
\end{aligned}
$$


[1] T. H. R. Skyrme, Proc. R. Soc. A 260, 127 (1961).

[2] G. S. Adkins, C. R. Nappi, and E. Witten, Nucl. Phys. B228, 552 (1983).

[3] G. S. Adkins and C. R. Nappi, Nucl. Phys. B233, 109 (1984).

[4] M. Bander and F. Hayot, Phys. Rev. D 30, 1837 (1984).

[5] K. F. Liu, J. S. Zhang, and G. R. E. Black, Phys. Rev. D 30, 2015 (1984).

[6] E. Braaten and J. P. Ralston, Phys. Rev. D 31, 598 (1985).
[7] R. Rajaraman, H. M. Sommermann, J. Wambach, and H. W. Wyld, Phys. Rev. D 33, 287 (1986).

[8] B. A. Li, K. F. Liu, and M. M. Zhang, Phys. Rev. D 35, 1693 (1987).

[9] J. L. Gervais, A. Jevicki, and B. Sakita, Phys. Rev. D 12, 1038 (1975).

[10] J. L. Gervais, A. Jevicki, and B. Sakita, Phys. Rep. 23, 281 (1976). 